\title{
Dos semblanzas del Dr. Orestes Nicholas Stavroudis
}

\section{Una breve semblanza Académica y laboral de Nick}

Orestes Nicholas (Nick) Stavroudis Mizner dejó de existir el pasado 18 de enero, a causa de una insuficiencia cardiaca que se complicó en sus últimos días con un derrame cerebral que afectó solo la zona motriz, postrándolo en cama pero conservó su lucidez de razonamiento.

Nick recibió la Licenciatura y la

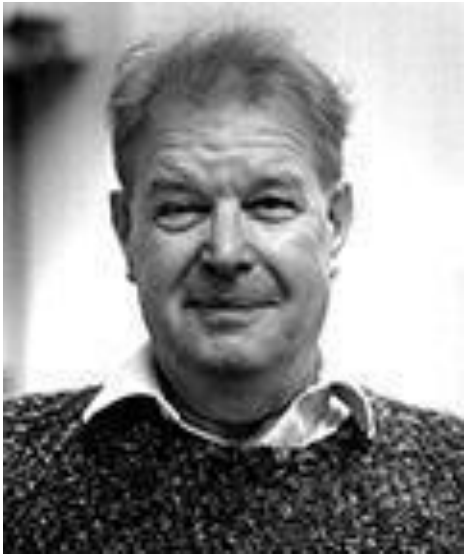

Mathematics; asi como de la legión de honor en ciencia y tecnología de Sigma Xi.

Su amplio sentido de colaboración y apoyo lo proyectó en la OSA. En la que participó como miembro organizador de programas, talleres, publicaciones y varios otros comités, uno de los más importantes fue el haber sido Editor Asociado de Journal of the Optical Society of America.
Maestría en matemáticas de la Universidad de Columbia, Estados Unidos. En 1959 el Dr. Stavroudis recibió su doctorado del Imperial College de la Universidad de Londres, Gran Bretaña, en donde él estudió óptica con el muy famoso Profesor W.T. Welford.

Nick trabajó como matemático por muchos años en el National Bureau of Standards de USA. Después pasó a laborar como profesorinvestigador en la facultad del Optical Sciences Center de la Universidad de Arizona en 1968.

Nick fue una persona ampliamente reconocida en el área del diseño óptico, así como por su destreza y habilidades en la Óptica Matemática. Su método de diseño modular fue uno de los más eficientes para sistemas muy complejos.

Su trabajo en la industria y en la Secretaría de la Defensa de Estados Unidos fue altamente reconocido. Después de haberse retirado de la Universidad de Arizona fue consultor privado. Trabajando en Lockeed obtuvo una patente por el Diseño de bafles reflectores para telescopios espaciales.

Stavroudis fue Fellow de la American Association for the Advancement of Science y de la Optical Society of America (OSA). También fue miembro de la Mathematical Society of America y de la Society for Industrial and Applied
Nick se incorporó a las actividades de investigación en el CIO a partir del $1^{\text {o }}$ de marzo de 1992. A través de un apoyo por parte del CONACYT, bajo Cátedra Patrimonial de Excelencia Nivel II desarrollando los siguientes proyectos:

-Diseño de sistemas telescópicos fuera de eje

- Comportamiento de frentes de onda geométricos durante la refracción

En sus últimos años en el CIO el Dr. Stavroudis estuvo publicando a un buen ritmo con el apoyo de sus entonces estudiantes Maximino Avendaño Alejo(hoy en CCADET-UNAM, México), Juan Hurtado Ramos (CICATA-IPN, Mexico), Gilberto Gómez Rosas (Univ. de Guadalajara), Ricardo Flores Hernández (CIO), entre otros.

Los intereses académicos de Nick incluyeron la geometría diferencial de frentes de onda, la función K (eikonal) y las ecuaciones de Maxwell, los telescopios "muy" fuera de-eje, el trazo generalizado de rayos y el diseño óptico modular.

Entre sus muchas publicaciones se encuentran varias monografías y tres libros. Una de las principales monografías que publicó Nick es "Spot Diagrams for the Prediction of Lens Performance From Design Data", publicado por el 


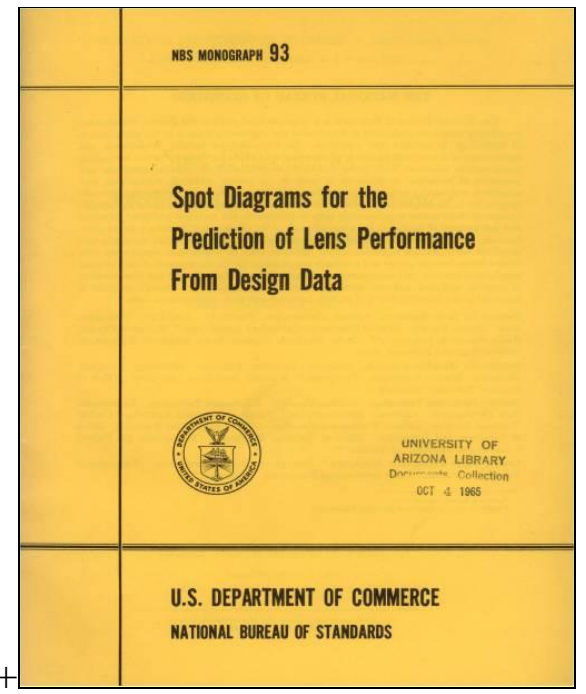

O.N. Stavroudis and Loyd E. Button, Spot Diagrams for the Prediction of Lens Performance From Design Data, published by US Department of Commerce, National Bureau of Standards, Monograph 93, Washington DC, 1965.

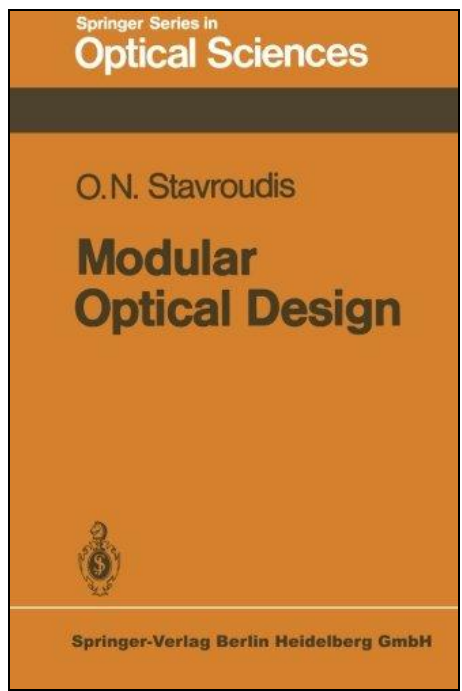

O.N. Stavroudis, Modular Optical Design, Springer-Verlag, Berlin, 1982

US Department of Commerce, National Bureau of Standards (hoy en dia se conoce como NIST), Monograph number 93 en 1965.

Sus tres libros, que son un referente y que actualmente son usados ampliamente en cursos formales de diseño óptico son:

- Optics of Rays, Wavefronts and Caustics, Academic Press Inc, New York, 1972.

- Modular Optical Design, Springer-Verlag, Berlin, 1982.

- The Mathematics of Geometrical and Physical Optics: The K-function and Its Ramifications, Wiley-VCH, Berlin, 2006.

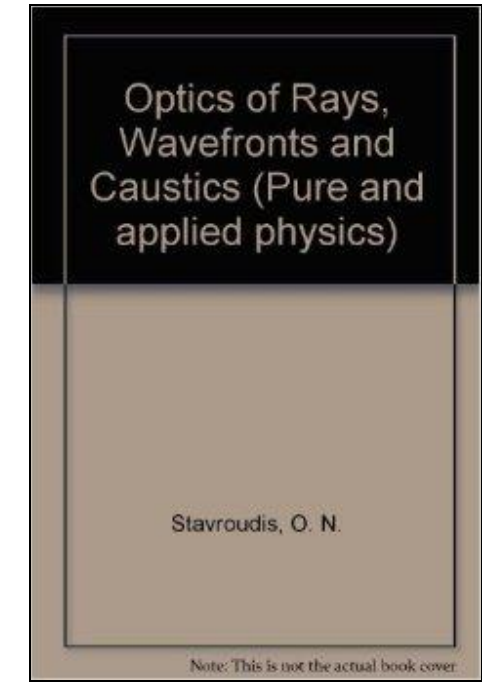

O.N. Stavroudis, Optics of Rays, Wavefronts and Caustics, Academic Press Inc, New York, 1972.

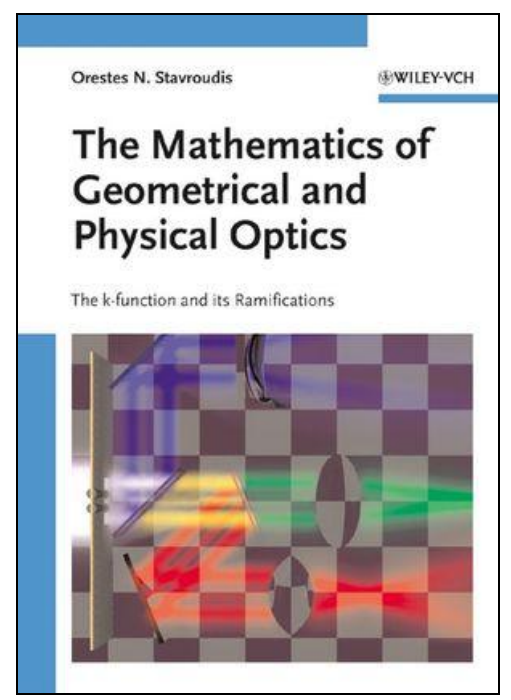

O.N. Stavroudis, The Mathematics of Geometrical and Physical Optics: The K-function and Its Ramifications, Wiley-VCH, 2006.

En la preparación de este último libro fue apoyado por su hijo Christopher y su estudiante "Maximiliano" (así le llamaba a Maximino Avendaño).

Toda la comunidad óptica nacional e internacional recordaremos con mucho afecto a nuestro colega Nick. Que en paz descanse.

\section{Ramón Rodríguez Vera,} Centro de Investigaciones en Optica, A.C. (CIO) y Presidente Academia Mexicana de Óptica (AMO) 2011-2012. 


\section{Breve semblanza histórico-anecdótica de Nick}

Yo fui alumno doctoral de Nick, lo que además de la ventaja académica me dio magnificas oportunidades de conocerle como persona. Paso a recordar algunos aspectos que me compartió de su vida, de su interacción conmigo y con el CIO.

Conocí a Nick en casa de Daniel Malacara. Hacía poco que se había jubilado del Optical Sciences Center (OSC, Tucson) y dado que su hijo menor Gregory estaba trabajando en Guadalajara, él y su esposa Dorle decidieron cambiarse allá.

Como era una persona muy activa, no podía estar sin hacer nada, decidió solicitar trabajo como profesor en la Universidad de Guadalajara.... y sí, se lo dieron: una clase de algebra elemental. Dado que León esta cerca, un buen día decidió venir a visitar a su amigo Daniel y ahí actuó el afortunado destino: Estando Nick con Daniel, con intensiones de "visita de doctor", llegó primero Arquímedes Morales y casi inmediatamente después llegué yo.

Cuando Daniel me lo presentó, mencionando que venía del OSC, yo le dije "Ah entonces Ud. conoce el método Y-Y barra". El sonrió y dijo "Es mi favorito" a lo que respondí "iVenga esa mano, Ud. es de los míos!". Ahí comenzó nuestra amistad. Se dio la usual conversación amigable, en ella Nick externó que la clase que le habían dado en Guadalajara, aunque le hacía una persona útil no le satisfacía plenamente. Entonces Daniel le dijo a Arquímedes “¿Por qué no le ofreces trabajo a Nick en el CIO?", a lo que Arquímedes contestó en forma afirmativa y en pocos días lo tuvimos aquí, colaborando con nuestro CIO.

La interacción entre nosotros inicialmente fue solo amistosa, especialmente por la cercanía de nuestros cubículos. Por esas breves conversaciones entré en contacto con su conocimiento casi enciclopédico de Historia; en lo único que yo le ganaba era en historia de México, especialmente de la etapa precolombina, pero aún en la historia de nuestro país me dio algunas sorpresas.

Por esas fechas vi la necesidad de completar mi formación académica con el Doctorado. Dada mi natural tendencia a las cosas prácticas, inicialmente le pedí a Raul Casas (Profesor visitante durante varios veranos y que fue Jefe del departamento de diseño en Perkin Elmer durante más de veinte años) que fuera mi asesor $\mathrm{y}$ comenzamos a trabajar. El proyecto que convenimos desarrollar era el diseño de una cámara satelital multiespectral, para colectar información de uso de suelos, recursos naturales, etc. y con una resolución de $50 \mathrm{~m} \times 50 \mathrm{~m}$ para que no fuera excesivamente cara y de paso que no tuviera "impedimentos estratégicos" de parte de las potencias extranjeras; eran los tiempos finales de la guerra fría. No pude formalizar el registro del proyecto, porque un director de tesis doctoral debía ser un Doctor, no un Maestro en ciencias (el grado académico de Raul).

La siguiente opción por supuesto que fue Nick, que aunque era Doctor en matemáticas, tenía mucha experiencia y también trabajos y libros publicados en Diseño óptico. Él estuvo de acuerdo y me dijo: "Piensa una propuesta de investigación en Diseño, para que la discutamos". Como el área común de intereses entre el y yo era el Método Y-Y barra y dado que ya había yo obtenido algunos resultados interesantes, le describí en forma muy breve el tema "Incrementos diferenciales de las aberraciones de Seidel en el espacio Y-Y barra".... y le hice un pequeño "show en computadora" consistente en un importante descenso de las aberraciones de un triplete Cooke mediante el simple desplazamiento de uno de los puntos del diagrama correspondiente. Cuando lo vió, exclamó "Son of a bitch!, that is great!". Con lo que el tema quedó aprobado y se iniciaron los trámites y el trabajo correspondiente. Obtuve mi doctorado en Noviembre de 1996. Tuve que hacer el programa de cómputo en el que puse en práctica mis resultados en dos versiones: a color y blanco y negro por un detalle que narro más adelante.

Durante los dos años de trabajo doctoral visité muchas veces a Nick y a su esposa Dorla en su casa, para tratar detalles del desarrollo del trabajo, a lo cual seguía una agradable convivencia degustando una copa de vino y una amable conversación, especialmente de aventuras vividas tanto por el como por mí. Aquí van algunas que recuerdo.

Nick fue un chico como casi todos, incluyendo el típico accidente en bicicleta en el que se fue a estrellar contra la cerca de un vecino, rompiéndola y prácticamente destruyendo su bicicleta. Como un tío suyo poseía unos barcos mercantes (el que insistió que lo bautizaran "Orestes"), obtuvo trabajo como grumete en su barco más pequeño, que hacía servicio de cabotaje transportando mercancía a lo largo de los puertos de la costa atlántica de EEUU y ganó algún dinero. 
Cuando Nick ingresó al Army Corps para su servicio militar, ocurrió el ataque Japonés a Pearl Harbor y Alemania le declaró la guerra a EEUU. Era necesario iniciar operaciones de defensa, especialmente para impedir que por medio de sus submarinos los alemanes ingresaran espías o saboteadores a EEUU y así el destacamento de Nick fue desplegado a lo largo de la costa de Florida, cada recluta convenientemente armado con jun bate de baseball!!!!.

Después ingresó a la fuerza aérea, entonces parte del ejército, recibió entrenamiento como artillero de cola de bombarderos "Liberator" y cuando estaba a punto de ser enviado al norte de África para desde ahí volar a atacar los campos petroleros de Rumania volando sobre Creta, Grecia y Yugoslavia ocupadas por los nazis.

En el último examen médico antes de enviarlo a esas misiones tan peligrosas descubrieron que Nick era ciego al color, daltónico al cien por ciento y entonces no lo enviaron, se quedó en la misma base para ser entrenador de los nuevos reclutas que llegaban continuamente. (Por eso la necesidad de la versión ByN de mi programa) Esto fue muy afortunado, porque de los artilleros de cola que si fueron a esas misiones solo el $10 \%$ regresaron, usualmente muy mal heridos, así Nick salvó la vida.

Permaneció como entrenador en esa base aérea (en Arizona) toda la guerra, capacitándose más y más, llegando a ser un experto en todos los armamentos de defensa y ataque de diversos tipos de bombarderos, hasta del B29, incluyendo la computadora analógica electrónica (del tamaño de un refrigerador) que dirigía la puntería de todas las torretas artilladas, cada una con cuatro ametralladoras calibre 0.50 .

En esos años interactuó con personas de todas las calidades, desde uno que le encantaba escaparse para montar a caballo por el desierto abandonando sus responsabilidades en la base, lo que enojaba mucho a Nick, y que (suertudo ese tipo) andando en una de esas escapadas le tocó estar cerca del sitio donde cayó un avión por fallas mecánicas y fue el primero en llegar a auxiliar a los heridos; por lo que le dieron una mención honorífica, lo que acabó de enojar a Nick.

$Y$ hasta otro que una vez, estando cargando bombas reales de $50 \mathrm{~kg}$ en un bombardero, para un ejercicio real de bombardeo de una tripulación en entrenamiento (Nick a cargo, arriba de una escalera y el otro tipo abajo), de repente uno de los dos sujetadores de la bomba se rompió y Nick la tuvo que cargar casi totalmente. El tipo le preguntó "Nick ¿la tienes bien agarrada?". Cuando Nick respondió "si", el otro pegó veloz carrera dejándolo solo cargando esa bomba "viva" y mal acomodado arriba de la escalera, hasta que después de un rato vinieron otros compañeros a auxiliarlo.

Ya hacia el final de la guerra cuando Alemania ya había sido vencida y quedaba solo Japón luchando, escaseaban los reclutas, se ordenó que todo personal de entrenamiento fuera enviado al Pacífico para participar en el asalto definitivo contra Japón, no como entrenadores, sino como combatientes reales, Nick empezó a preparar sus cositas para ese viaje y para esas misiones de bombardeo volando entre Kamikazes que peleaban furiosamente; entonces ocurrieron los bombardeos atómicos de Hiroshima y Nagasaki y la guerra terminó.

Nick fué desmovilizado de inmediato y como veterano de guerra recibió el beneficio de una beca para estudiar lo que quisiera.... y él escogió Matemáticas, obteniendo la Maestría de la Columbia University en Nueva York NY. Ahí en NY conoció a la que muy pronto fue su esposa: Dorla. Ya casados y todavía con el apoyo del gobierno federal de EEUU por ser veterano de guerra, fue a hacer el doctorado en matemáticas al Imperial College de Londres. Cuando se presentó ante el que fue su asesor, se asomó por la ventana y vio en el jardín un hermoso laguito circular, y dijo "Que hermoso lago" a lo que el profesor le contestó "No existía antes de la guerra, lo hizo una bomba V2 alemana".

Una vez obtenido el grado de Doctor y como no habían disfrutado de un viaje de luna de miel, decidieron realizar un paseo por Europa, a bordo de una motocicleta de tercera mano que compraron por un módico precio, pero que se descomponía cada pocos días. Recorrieron Francia $\mathrm{y}$ no entraron a Alemania por los amargos recuerdos que conservaba Dorla (había emigrado a EEUU con su familia durante la guerra). Pasando Suiza entraron a Austria, el país natal de Dorla, de donde se había escapado caminando hacia Suiza junto con sus padres y sus dos hermanas pocos días antes de la "unión" forzada de Austria a Alemania. Llegando a Viena sobre su vetusta moto quedaron impresionados por el despliegue de fuerza de los rusos que eran ahí el ejército de 
ocupación de ese anterior miembro del Reich alemán. Comenzaba la guerra fría y parecía que en cualquier momento se podría poner caliente, así que decidieron poner fin a su viaje, vendieron la moto y regresaron a EEUU.

Pronto Nick obtuvo trabajo en el National Bureau of Standards (NBS), ahora llamado NIST en el departamento de pruebas ópticas, para que aplicara sus conocimientos matemáticos en ese campo. Ahí conoció a Roland V. Shack y se hicieron grandes amigos. La función de ese departamento no era precisamente el diseño de sistemas ópticos, sino el de evaluar la calidad de los prototipos entregados por la industria a diversas dependencias de gobierno, especialmente las tres fuerzas armadas.

Un caso interesante ocurrió cuando una compañía entregó una cámara para fotografía aérea de alta resolución a una de las armas, cuyo prototipo funcionaba de maravilla (hecho por esa compañía), pero cuyas réplicas hechas por la dependencia armada en base a las especificaciones recibidas, funcionaban de lo peor, muy mal. Entonces la dependencia solicitante envió al NBS dos ejemplares: el prototipo y una de las réplicas que habían fabricado, junto con toda la documentación de diseño óptico y mecánico para que el NBS determinara si el proveedor había incumplido el contrato de alguna forma. El análisis fue un verdadero rompecabezas, todo parecía ajustarse a las especificaciones y a los planos de diseño, pero la réplica seguía siendo un caso perdido; así que entonces decidieron desarmar la cámara prototipo para ver si algo era diferente. Primero verificaron la mecánica (todo $\mathrm{OK}$ ) y al final de un día de trabajo, con las componentes ópticas colocadas sobre una mesa, al pasar Nick vio algo raro: la reflexión de las lámparas del techo sobre una de las componentes ópticas aparecía extrañamente deformada, se lo comentó a sus compañeros de equipo y al día siguiente enfocaron su estudio sobre esa pieza: una de sus superficies NO era esférica, sino asférica, de orden superior, no una cónica. Cargando esos datos en computadora y trazando rayos todo checaba, los cálculos coincidían con el comportamiento real del prototipo. Los documentos entregados no mencionaban nada acerca de asfericidades así que se llamó a los representantes de la compañía responsable del diseño y del prototipo y a representantes de la fuerza armada. Les preguntaron a los proveedores del prototipo porqué no habían entregado la información completa, a lo que respondieron: "Señores, el contrato especifica que debíamos entregar todos los radios de curvatura, verifiquen el radio de curvatura central de esa superficie y comprobarán que es el indicado en los planos, no pusimos los coeficientes de asfericidad porque el contrato no lo especifica". En vez de iniciar un pleito legal, los representantes de la fuerza armada decidieron dar por concluido el asunto y encargaron el proyecto a otra compañía, con una redacción mucho más cuidadosa que en esa ocasión.

Las cosas fueron bien y tranquilas en el NBS hasta que ocurrió un cambio de administración seguido de una reorganización de departamentos que no fue del agrado de Roland Shack, renunció y se fue al recién fundado Optical Sciences Center (OSC). Nick aguantó un poco más pero al poco tiempo la situación se volvió insoportable, renunció y también se fué al OSC, donde trabajó hasta alcanzar la edad de jubilación, publicando muchos papers, varios libros y dirigiendo tesis de Maestría y Doctorado de profesionistas que aún trabajan en Diseño Óptico, por ejemplo Romeo Mercado (Filipino) que ha trabajado para diversas compañías Japonesas y americanas; Actualmente Romeo trabaja en Apple, diseñando las lentes de sus dispositivos ("gadgets") mediante el método que fue su tema de tesis doctoral bajo la dirección de Nick: Modular Optical Design, una aplicación particular del método Y-Y barra.

Incapaz de quedarse inactivo, Nick se fue a California y entró a trabajar a Lockeed, que deseaba "comprimir" más instrumentos ópticos en cada satélite. El problema era conectar diversos instrumentos ópticos a un solo telescopio y que todo el sistema fuera menos pesado y más eficiente. Nick propuso colocar los instrumentos en una estructura anular alrededor del espejo primario, con un espejo "terciario" central giratorio que enviara la imagen al experimento en turno. Como cada dispositivo debía armarse dentro de un segmento de cilindro, las componentes deberían ser "Shiefspiegler" (espejos muy fuera de eje) para cumplir simultáneamente dos funciones: tener poder óptico y desviar la trayectoria del haz según la geometría cilíndrica del espacio disponible. Eso abrió las puertas para que Nick volviera a trabajar 


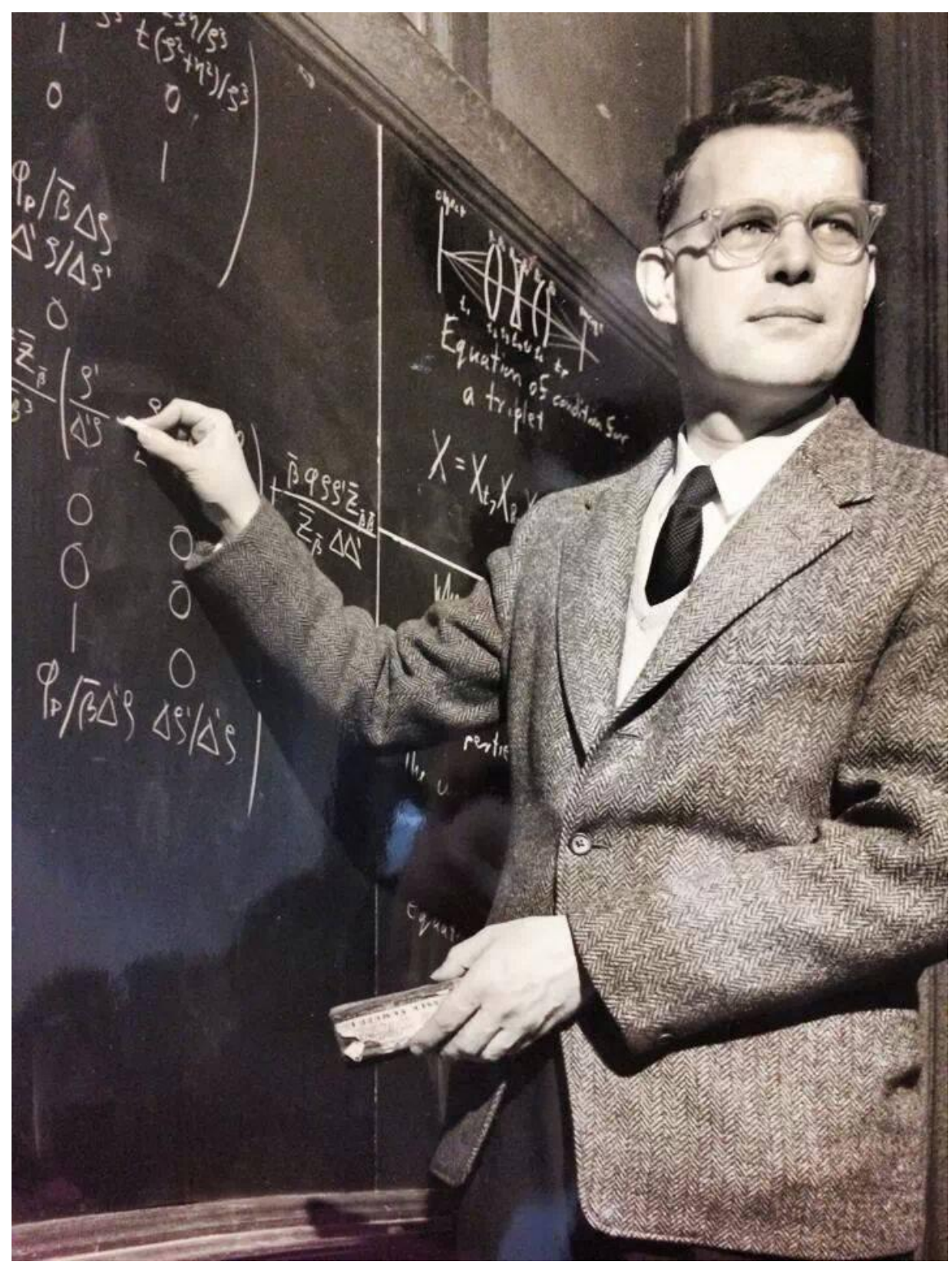

Fig. 1. Nick en el NBS (National Bureau of Standards), Washington. Explicando los errores de propagación de rayos mediante tensores. Note el ejemplo: un triplete Cooke.

con un "viejo amor", la óptica de los espejos fuertemente fuera de eje, en la que trabajó también en el CIO, publicando desde aquí dos o tres papers sobre el tema.

El otro aspecto que aumentaba mucho el peso de los satélites eran los "baffles" que impiden la entrada de luz no deseada al sistema. Nick diseñó y obtuvo patente por un sistema de baffles totalmente reflectivos que funcionan mejor que los absortivos y que al no introducir calor al sistema lo hacen funcionar mejor y de manera más larga y confiable.
Pero lamentablemente (o afortunadamente) terminó la guerra fría, se recortaron drásticamente los presupuestos, se cancelaron proyectos y empezó el despido de personal. Los primeros en ser despedidos fueron los técnicos, luego los ingenieros, luego los científicos (entre ellos Nick); luego el personal administrativo; al final quedaron solo los jefes y sus secretarias (jinteresante orden de prioridades!). En ese momento fue cuando Nick y Dorla decidieron mudarse a Guadalajara y el subsiguiente ingreso de Nick al CIO. 
Otro campo en el que Nick hizo aportaciones valiosas fue la Función Eikonal, que el llamó "The K-function", que se puede resumir diciendo que consiste en el diseño de sistemas ópticos planteando su comportamiento exclusivamente por medio del Principio de Fermat; trabajo muy complicado, inconcluso todavía hoy y tal parece que continuará así por algún tiempo más, por la gran complejidad que involucra y el escaso número de "voluntarios" deseosos de atacar dicho problema.

Nick ya no está con nosotros, descansa en paz después de una vida llena de aventuras y grandes contribuciones a la ciencia y tecnología que nosotros también amamos: la Óptica.

Ricardo B. Flores Hernández, Centro de Investigaciones en Optica, A.C.

DOI: http://dx.doi.org/10.7149/OPA.47.1.71 